\title{
Treatment of Nanophthalmos-Related Uveal Effusion with Two- vs. Four-Quadrant Partial-Thickness Sclerectomy and Sclerotomy Surgery
}

(D) Şengül Özdek*, (1) Duygu Yalınbaş Yeter**, (1) Mehmet Cüneyt Özmen*, (D Murat Hasanreisoğlu*

*Gazi University Faculty of Medicine, Department of Ophthalmology, Ankara, Turkey

**Sivas Cumhuriyet University Faculty of Medicine, Department of Ophthalmology, Sivas, Turkey

\begin{abstract}
Objectives: To report visual and anatomical outcomes following two- or four-quadrant partial-thickness sclerectomy and sclerotomy surgery to treat nanophthalmos (NO)-related uveal effusion (UE).

Materials and Methods: Consecutive patients with NO-related UE were treated with four-quadrant or two-quadrant (for those with associated glaucoma) partial-thickness sclerectomy and sclerotomy surgery. Axial length, extent of UE, preoperative, postoperative, and final best corrected visual acuity (BCVA), time to retinal reattachment, and rates of retinal reattachment and recurrence were noted. Results: Fourteen eyes of 10 patients with NO-related UE were operated. Retinal detachment (RD) involved mainly the peripheral retina in 7 (50\%) eyes, macula in 2 eyes (14.2\%), both macula and peripheral retina in 4 eyes (28.6\%), and the whole retina in 1 eye. Eleven eyes had four-quadrant surgery, and 3 eyes with associated glaucoma had two-quadrant surgery. External subretinal drainage was performed in one patient who had total RD. The mean preoperative $\log$ MAR BCVA of $1.50 \pm 0.53$ increased significantly to $0.92 \pm 0.49$ after surgery ( $\mathrm{p}=0.002)$. Resolution of RD could be achieved with two-quadrant surgery in only 1 of 3 eyes. In the other 2 eyes, retinal reattachment was achieved after a secondary surgery for the remaining two quadrants to complete four-quadrant sclerectomy. Final outcome was total reattachment of the retina in 11 eyes (78.6\%), partial reattachment in 1 eye $(7.1 \%)$, and recurrence of macular detachment in $2(14.3 \%)$ eyes.

Conclusion: Quadrantic partial-thickness sclerectomy and sclerotomy surgery seems effective for treating UE in eyes with NO. Twoquadrant surgery may be tried for mild UE associated with glaucoma to preserve the superior quadrants for future possible glaucoma surgeries, but secondary surgery for the superior quadrants may be needed. External drainage of subretinal fluid may be an option in severe cases to achieve quicker resolution.
\end{abstract}

Keywords: Nanophthalmos, partial sclerectomy, sclerotomy, uveal effusion, exudative retinal detachment

Address for Correspondence: Şengül Özdek, Gazi University Faculty of Medicine, Department of Ophthalmology, Ankara, Turkey E-mail: sengulozdek@gmail.com ORCID-ID: orcid.org/0000-0002-7494-4106

Received: 16.12.2020 Accepted: 26.03.2021

Cite this article as: Özdek Ş, Yalnıbaş Yeter D, Özmen MC, Hasanreisoğlu M. Treatment of Nanophthalmos-Related Uveal Effusion with Two- vs. Four-Quadrant Partial-Thickness Sclerectomy and Sclerotomy Surgery. Turk J Ophthalmol 2022;52:37-44

${ }^{\oplus}$ Copyright 2022 by Turkish Ophthalmological Association Turkish Journal of Ophthalmology, published by Galenos Publishing House. 


\section{Introduction}

Nanophthalmos (NO) comes from the Latin word nanos, which means "dwarf." $\mathrm{NO}$ is thought to result from developmental arrest of the globe. In contrast to microphthalmos, which is frequently complicated by developmental defects of the fetal fissure and coloboma of the iris, choroid, and retina, the nanophthalmic eye is typically reduced in volume with a normal-sized crystalline lens, resulting in a high lens to eye volume ratio. ${ }^{1}$

The incidence of $\mathrm{NO}$ was reported to range from $0.002 \%$ to $0.017 \%$ in a British cohort. ${ }^{2}$ Clinical features include a deepset globe within a small orbit with narrow palpebral fissures, short axial length $(<20.5 \mathrm{~mm})$, axial hypermetropia $(+8.00$ to +20.00$)$, a shallow and crowded anterior chamber, and thickened sclera. ${ }^{3,4}$ The thickened and abnormal sclera causes angle-closure glaucoma and posterior segment problems such as uveal effusion (UE), macular hypoplasia, chorioretinal folds, pseudopapilledema, and retinal pigment epithelium (RPE) hyperpigmentation. ${ }^{5}$

UE may develop spontaneously or after an uncomplicated anterior segment surgery such as cataract surgery. ${ }^{6}$ Different theories regarding the pathophysiology of UE have been speculated, including vortex vein compression, choroidal permeability changes, and decreased scleral permeability. ${ }^{7}$ Gass $^{8}$ proposed that subretinal fluid may be absorbed transsclerally instead of through vortex veins and suggested that UE develops not as a result of vortex vein compression, but because the thickened and abnormal sclera blocks transscleral fluid absorption. He also suggested that when the sclera is thinned with sclerectomies, effusion may regress. It was reported that resorption of the subretinal fluid could be achieved with sclerectomies alone in a group of patients with UE. ${ }^{9}$ Vortex vein decompression, partial-thickness sclerectomy, partial-thickness sclerectomy with mitomycin C (MMC), partial-thickness sclerectomy with incision or punch sclerostomy, and subretinal fluid drainage are some of the reported procedures to treat this condition. ${ }^{10}$

However, glaucoma is another significant problem encountered in eyes with NO. ${ }^{11}$ When partial-thickness sclerectomy and sclerotomy surgery is performed in four quadrants, glaucoma surgery would be very problematic and aggressive when needed later in life because of the scarred conjunctiva. Therefore, an inferior two-quadrant surgery in eyes with glaucoma may be a good option to protect the superior quadrants from surgical damage.

The aim of our study was to report visual and anatomical outcomes after two- or four-quadrant partial-thickness sclerectomy and sclerotomy surgery for the treatment of NO-related UE.

\section{Materials and Methods}

This retrospective, non-comparative, consecutive interventional case series included patients with NO-related UE. The design of the study was approved by the local ethics committee of the university. The study protocol conformed to the ethical guidelines of the 1975 Declaration of Helsinki, and all participants gave written informed consent. The patients' records were reviewed to obtain complete ophthalmic examination findings including best corrected visual acuity (BCVA), slitlamp examination, intraocular pressure (IOP) measurement, fundus examination, optical coherence tomography (OCT), and axial length (AL) measured with A-scan ultrasonography. Patients with glaucoma underwent inferior two-quadrant, and all others underwent four-quadrant partial-thickness sclerectomy and sclerotomy surgery.

Surgical technique (Figure 1): All surgeries were done by three experienced retinal surgeons (S.O., M.C.O., and M.H.) under retrobulbar anesthesia. Briefly, the technique involved the creation of rectangular partial-thickness (two-thirds to three-quarters) sclerectomies measuring approximately $6 \times 4 \mathrm{~mm}$ (depending on the size of the globe) in the two inferior quadrants or all four quadrants. The anterior boundary was the rectus muscle insertions, the posterior boundary was the vortex vein ampulla to avoid its intrascleral course, and the lateral boundary was the rectus muscles, leaving a very narrow strip of sclera blocked by the muscle mass. The superior oblique muscle insertion area was spared in the superotemporal quadrant to avoid damage. First, a partial-thickness scleral incision was made to delineate the extent and depth of the scleral dissection (Figure 1a). Partial-thickness scleral flaps approximately $80-90 \%$ of the original thickness were dissected and removed (Figure 1b,c). A linear sclerotomy was created in the center of the sclerectomy area to determine the thickness of remaining scleral until the choroid is seen (Figure 1d). No attempt was made to drain the subretinal fluid (except in 1 eye) or decompress the vortex veins.

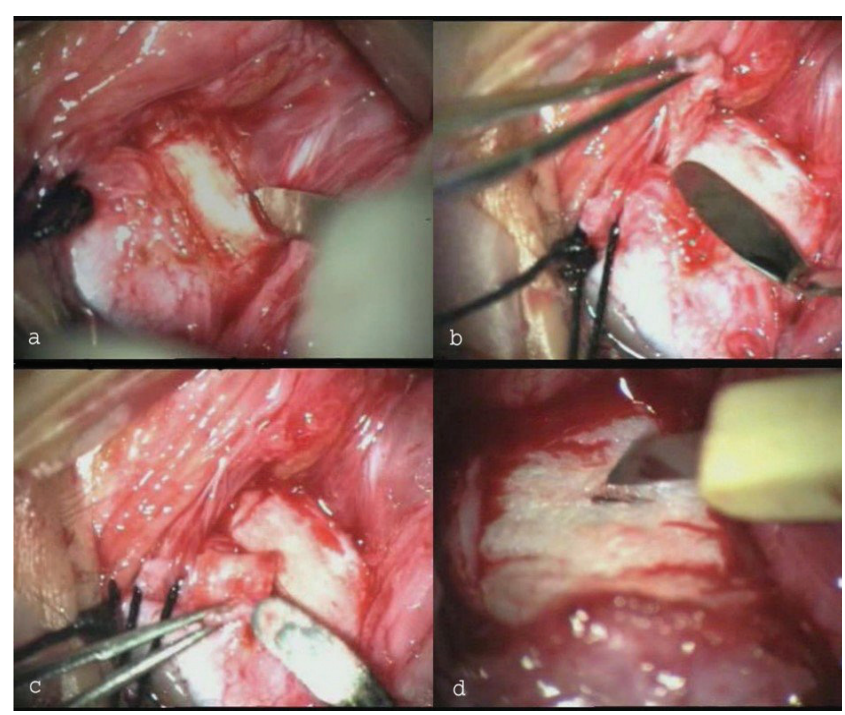

Figure 1. a) Partial-thickness rectangular scleral incision is made to delineate the scleral dissection. b, c) Dissection of the scleral flap to be removed. d) Linear sclerotomy in the center of the sclerectomy area 
Surgery was performed only in the inferior two quadrants in 3 eyes of 3 patients with known glaucoma to preserve the superior conjunctiva and sclera for glaucoma surgery that may be needed in the future. Drainage of subretinal fluid was performed only in 1 eye with the most severe UE using a 20-gauge MVR knife after cauterizing the choroid in the superotemporal quadrant. The eye was infused with balanced salt solution (BSS) through a limbal incision during external drainage.

Postoperative control visits were performed at 2 weeks, 1 , 3, 6, and 12 months, and every 6 months thereafter. Outcomes were classified as total reattachment of the retina if the effusions had resorbed entirely within 3 months, partial reattachment if there was a reduction in the subretinal fluid over 3 months, and unsuccessful if the fluid collections failed to improve within 3 months of surgery. A secondary surgery with superior quadrant sclerectomies was suggested to patients who underwent primary inferior two-quadrant sclerectomy if resolution of the subretinal fluid was not observed in the first 3 months postoperatively. Reoperation with mitomycin C (MMC) was performed in eyes that underwent primary four-quadrant sclerectomy if subretinal fluid increased during follow-up.

\section{Statistical analysis}

Data obtained in the study were analyzed statistically using SPSS for Windows version 20 (IBM Corp., Armonk, NY, USA). Values were summarized using mean \pm standard deviation or median (minimum-maximum) according to data distribution. Categorical variables were presented as number (n) and percentage (\%). Wilcoxon test was used to compare preoperative and postoperative BCVA. A value of $\mathrm{p} \leq 0.05$ was considered statistically significant.

\section{Results}

Fourteen eyes of 10 patients were included in the study. Demographic features of the patients are shown in Table 1. There were 7 male patients and 3 female patients, and the mean age was $32.7 \pm 14.1$ (14-64) years. Four of the 10 patients had bilateral surgery. The mean AL of 14 eyes was $15.1 \pm 2.7 \mathrm{~mm}$ $(10.2-20.1 \mathrm{~mm})$. The mean refractive error was $+15.0 \pm 3.9$ diopters (D) (+8.00-+18.25 D). Nine right eyes and 5 left eyes underwent surgery. Seven $(50.0 \%)$ of 14 eyes had peripheral retinal detachment (RD), 1 patient had bilateral (14.3\%) severe (highly elevated) localized macular RD with pigmentary retinopathy, 4 of 14 eyes (28.6\%) had both macular and peripheral RD, and 1 eye had total RD. Four of 14 eyes (28.6\%) had glaucoma. Two patients had pseudophakia with piggyback IOLs (operated elsewhere), and one of them underwent pars plana vitrectomy (PPV) and intravitreal silicone oil tamponade surgery for macular hole later (Table 1). Case 1 and 2 were brother and sister whose father also had NO (case 10).
Eleven eyes had four-quadrant surgery, and 3 eyes with known glaucoma (case 5, case 6, and case 10) had twoquadrant surgery. Case 5 gradually resolved within 12 weeks with only two-quadrant surgery. Case 6 , however, showed a transient partial resolution and required a second operation at postoperative 4 months to complete four-quadrant surgery, which resulted in total resolution of the subretinal fluid within 3 months. Case 10 also needed surgery in the other two quadrants at postoperative 8 months. This patient had highly elevated retinoschisis in the temporal peripheral retina, sparing the macula, which was demarcated with laser photocoagulation later following total resolution of the UE. The median follow-up after the surgery was 32.5 months (range 5-96 months). Final outcome was total reattachment of the retina in 11 eyes (78.6\%), partial reattachment in 1 eye $(7.1 \%)$, and recurrence in 2 eyes $(14.3 \%)$. The mean time to resolution after surgery was $8.4 \pm 7.1$ weeks (range 1-20 weeks).

The mean preoperative BCVA in logarithm of the minimum angle of resolution ( $\log$ MAR) units was $1.50 \pm 0.53$, which significantly increased to $0.92 \pm 0.49 \log$ MAR postoperatively at the end of follow-up ( $\mathrm{p}=0.002)$. BCVA improved in 12 eyes $(85.7 \%)$ and remained stable in 2 eyes $(14.3 \%)$.

Case 3: A 14-year-old boy presented with BCVA of 20/400 with $+17.00 \mathrm{D}$ correction bilaterally. He had bilateral highly elevated macular detachment with peripheral pigmentary retinopathy. Following bilateral four-quadrant partial-thickness sclerectomy and sclerotomy surgery, BCVA improved to 20/200 in both eyes with an evident subjective increase in visual quality within 5 months. Fundus examination and OCT demonstrated reduction in macular detachment height with partial resolution of submacular fluid. However, his vision deteriorated again to the preoperative level with an increase in macular UE height at 9 months. Episcleral scarring was thought to be the reason for the clinical worsening. Thus, he was reoperated with MMC as suggested by Akduman et al. ${ }^{12}$ The sclera was seen to be surprisingly thickened with fibrosis during the second surgery and was resected in the same way as in the first surgery. During postoperative follow-up, the subretinal fluid decreased minimally (not as much as after the first surgery).

Case 6: A 36-year-old woman had BCVA of 20/400 in her right eye and 20/60 in her left eye with $+15.00 \mathrm{D}$ correction and AL of 16.2 and $16.4 \mathrm{~mm}$, respectively. She had previously undergone YAG laser iridotomy for narrow-angle glaucoma elsewhere. IOP was $14 \mathrm{mmHg}$ without medication. She had foveal and peripheral RD in the right eye and peripheral limited $\mathrm{RD}$ in the left eye. We opted to perform two-quadrant (inferior) partial-thickness sclerectomy and sclerotomy surgery for the right eye to preserve the superior quadrant for the possible future need for glaucoma surgery. RD regressed partially during the first postoperative month but recurred over the next 4 months. 
Turk J Ophthalmol 52; 1: 2022

\begin{tabular}{|c|c|c|c|c|c|c|c|c|c|c|c|c|c|c|c|c|}
\hline & 鄢 & 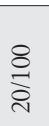 & $\stackrel{8}{\stackrel{1}{\grave{~}}}$ & $\stackrel{8}{\stackrel{2}{*}}$ & 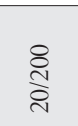 & $\begin{array}{l}\stackrel{\mathrm{N}}{ } \\
\stackrel{\text { ปे }}{ }\end{array}$ & $\underset{\underline{E}}{\widehat{E}}$ & ֻั & ֻั & 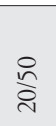 & $\underset{\text { छ्छ }}{\widehat{E}}$ & $\stackrel{8}{\stackrel{1}{2}}$ & 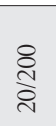 & $\stackrel{8}{\stackrel{\sim}{1}}$ & 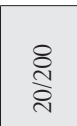 & 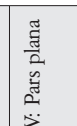 \\
\hline & 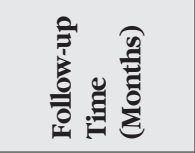 & 8 & $\stackrel{n}{n}$ & $\curvearrowright$ & 은 & 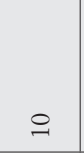 & ฉ & 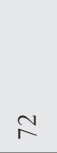 & in & $n$ & $\overleftrightarrow{\sim}$ & i & নे & 은 & $\stackrel{n}{2}$ & 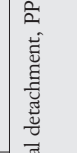 \\
\hline & 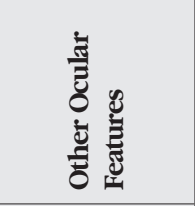 & & & & 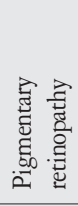 & 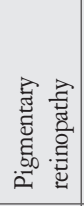 & 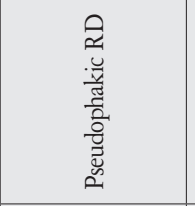 & 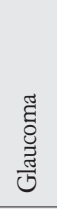 & 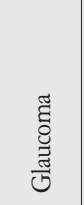 & 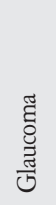 & 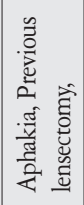 & 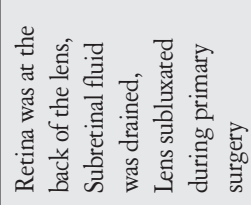 & 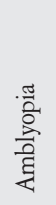 & 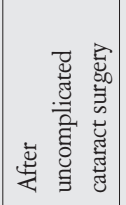 & 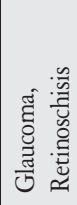 & 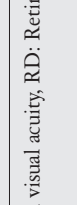 \\
\hline & 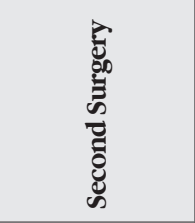 & . & , & ' & 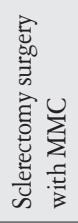 & 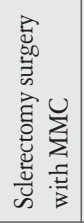 & 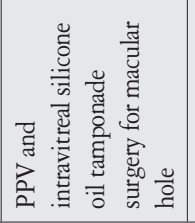 & & 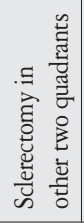 & & & 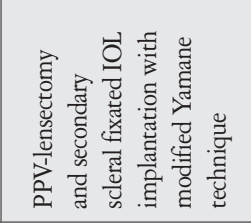 & , & & 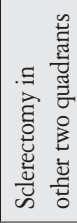 & 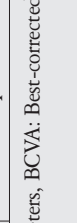 \\
\hline & 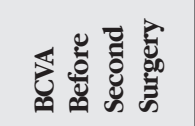 & & & & ڤั & 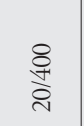 & 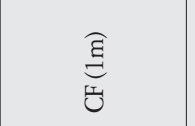 & & 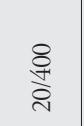 & & & ֻั & & & ষ্ণ & 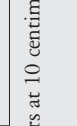 \\
\hline & 莺 & I & ક & Ð & I & I & $\frac{1}{ \pm}$ & £ & 玉 & ङ & £ & 玉 & Ð & I & モ & 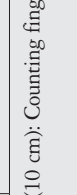 \\
\hline & 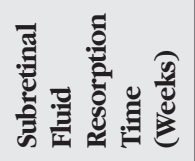 & $\sim$ & $N$ & $n$ & . & . & i & $\simeq$ & $\simeq$ & $n$ & $\checkmark$ & - & $\stackrel{\circ}{-}$ & $\checkmark$ & $\stackrel{\sim}{.}$ & 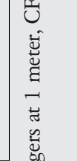 \\
\hline 节 & 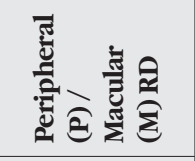 & $\stackrel{+}{ \pm}$ & $a$ & a & $\Sigma$ & $\Sigma$ & a & $\stackrel{+}{\sum_{2}^{+}}$ & $\stackrel{2}{\sum_{2}}$ & a & 2 & ङ & $\stackrel{+}{\sum_{2}^{+}}$ & 2 & 2 & 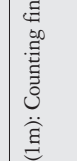 \\
\hline 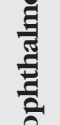 & 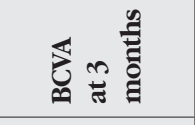 & 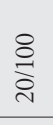 & 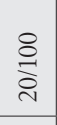 & ¿ั̀ & $\begin{array}{l}\text { ते } \\
\text { ڤે }\end{array}$ & ষ্ণ & 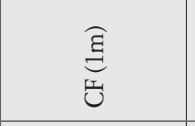 & 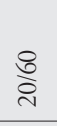 & ֻั & 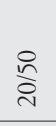 & 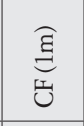 & ¿্ণ & ¿্̀ & 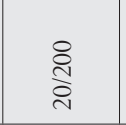 & ¿ั̀ & 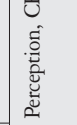 \\
\hline 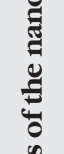 & 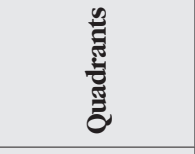 & $\forall$ & $\checkmark$ & 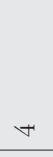 & $\forall$ & $\forall$ & $\forall$ & $\sim$ & N & $\forall$ & $\nabla$ & $\forall$ & $\forall$ & $t$ & $\sim$ & 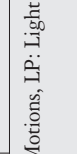 \\
\hline 离 & 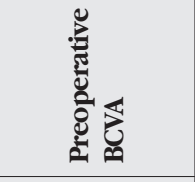 & $\stackrel{8}{\stackrel{+}{2}}$ & $\begin{array}{l}8 \\
\text { İ } \\
\end{array}$ & ষ্ণ & \begin{tabular}{l}
8 \\
$\stackrel{+}{+}$ \\
\multirow{2}{*}{}
\end{tabular} & 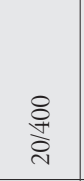 & 豈 & 氖 & 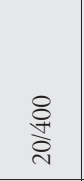 & $\stackrel{8}{\stackrel{2}{2}}$ & 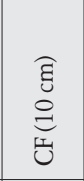 & $\Leftrightarrow$ & 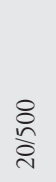 & 齐 & 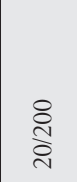 & 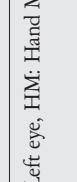 \\
\hline 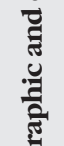 & 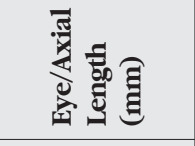 & 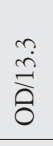 & 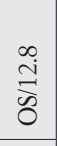 & $\underset{\mathscr{0}}{\stackrel{\overrightarrow{0}}{4}}$ & $\hat{\hat{े}}$ & $\frac{1}{3}$ & $\underset{\hat{\sigma}}{\tilde{0}}$ & $\begin{array}{l}\overrightarrow{\mathrm{N}} \\
\text { 0ิ }\end{array}$ & $\begin{array}{l}\widetilde{\sigma} \\
\stackrel{1}{0}\end{array}$ & $\frac{\vec{b}}{\stackrel{5}{8}}$ & $\stackrel{\stackrel{+}{*}}{\stackrel{0}{0}}$ & 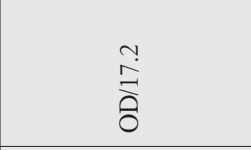 & $\begin{array}{l}\stackrel{0}{2} \\
\vec{\nabla}\end{array}$ & $\stackrel{\overrightarrow{0}}{\stackrel{\overbrace{}}{2}}$ & $\begin{array}{l}\stackrel{0}{0} \\
\stackrel{\overrightarrow{0}}{0}\end{array}$ & 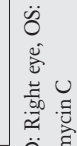 \\
\hline 气̆ & 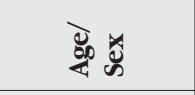 & 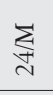 & $\sum_{i}^{\sum}$ & 崩 & $\underset{ \pm}{\Sigma}$ & $\underset{\Xi}{ \pm}$ & $\underset{\mathrm{i}}{\Sigma}$ & $\stackrel{\text { Lit }}{\vec{n}}$ & 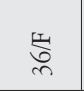 & Бे & $\underset{m}{\Sigma}$ & $\underset{\sim}{\Sigma}$ & $\underset{\sim}{\Sigma}$ & $\underset{\infty}{\Sigma}$ & $\underset{\mathrm{i}}{\sum}$ & בี \\
\hline 产 & 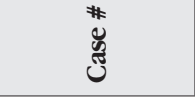 & - & & $N$ & $n$ & & $\checkmark$ & n & 0 & & $r$ & $\infty$ & & $a$ & $\stackrel{\circ}{=}$ & $\begin{array}{l}\sum_{z} \\
\end{array}$ \\
\hline
\end{tabular}


Another surgery was performed on the other two (superior) quadrants, which resulted in regression of the UE starting in the first postoperative days and total resolution at 12 weeks. Her BCVA improved to 20/60 in the right eye during the 50-month follow-up period. Three years later, the peripheral RD in the left eye progressed, and a four-quadrant (instead of two-quadrant) surgery was preferred because of our experience with her right eye. UE regressed totally in 5 weeks, and her BCVA improved to 20/50 in the left eye within 5 months of follow-up.

Case 8: A 32-year-old man presented with complaints of reduced vision in his right eye for 1.5 years and in his amblyopic left eye for 5 years. His BCVA was only light perception in his right eye, which used to be the better eye, and 20/500 in his left eye with +8.00 correction, and $\mathrm{AL}$ was approximately 17 $\mathrm{mm}$ bilaterally. He had leukocoria and the retina was at the back of the lens in his right eye (Figure 2a), and his left eye showed subtotal foveal and superior peripheral RD (Figure $2 \mathrm{~b})$. The right eye was thought to be inoperable, and a fourquadrant partial-thickness sclerectomy and sclerotomy surgery was performed on his left eye. Following surgery, UE regressed on postoperative day 1 and total reattachment was observed at 1 year (Figure 3). BCVA improved to 20/200 in the left eye. Eight months later, the patient requested treatment for his right eye and underwent four-quadrant partial-thickness sclerectomy and sclerotomy with subretinal fluid drainage for his right eye. After drainage of the subretinal fluid, the globe was repressurized with BSS through a limbal incision during the surgery. The retina moved posteriorly with drainage, but so did the crystalline lens. The retina totally reattached starting on postoperative day 1 . The dislocated lens in the vitreous cavity was removed with PPV/ lensectomy, and scleral-fixated IOL implantation was done using the modified Yamane technique in the right eye (Figure 4a). At last follow-up at 29 months, the retinas were totally reattached bilaterally and BCVA was 20/100 in the right eye and 20/200 in the left eye (Figure 4b).

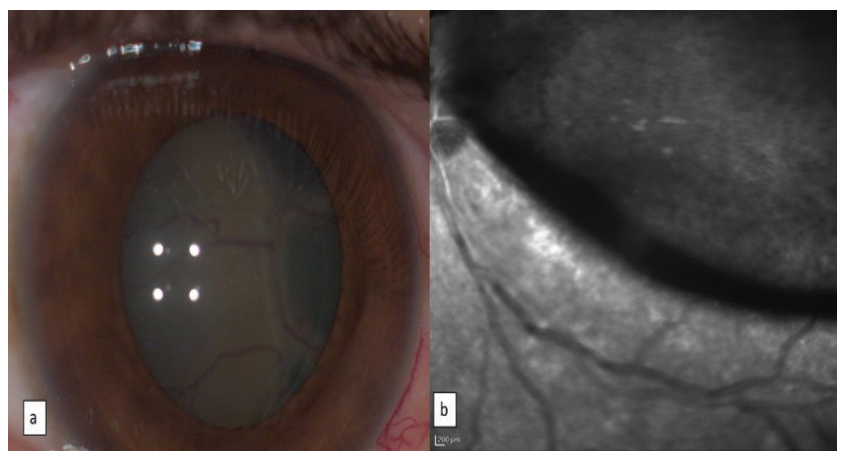

Figure 2. Case 8 had leukocoria with the retina at the back of the lens in his right eye (a) and subtotal foveal and peripheral exudative detachment in his left eye (b)

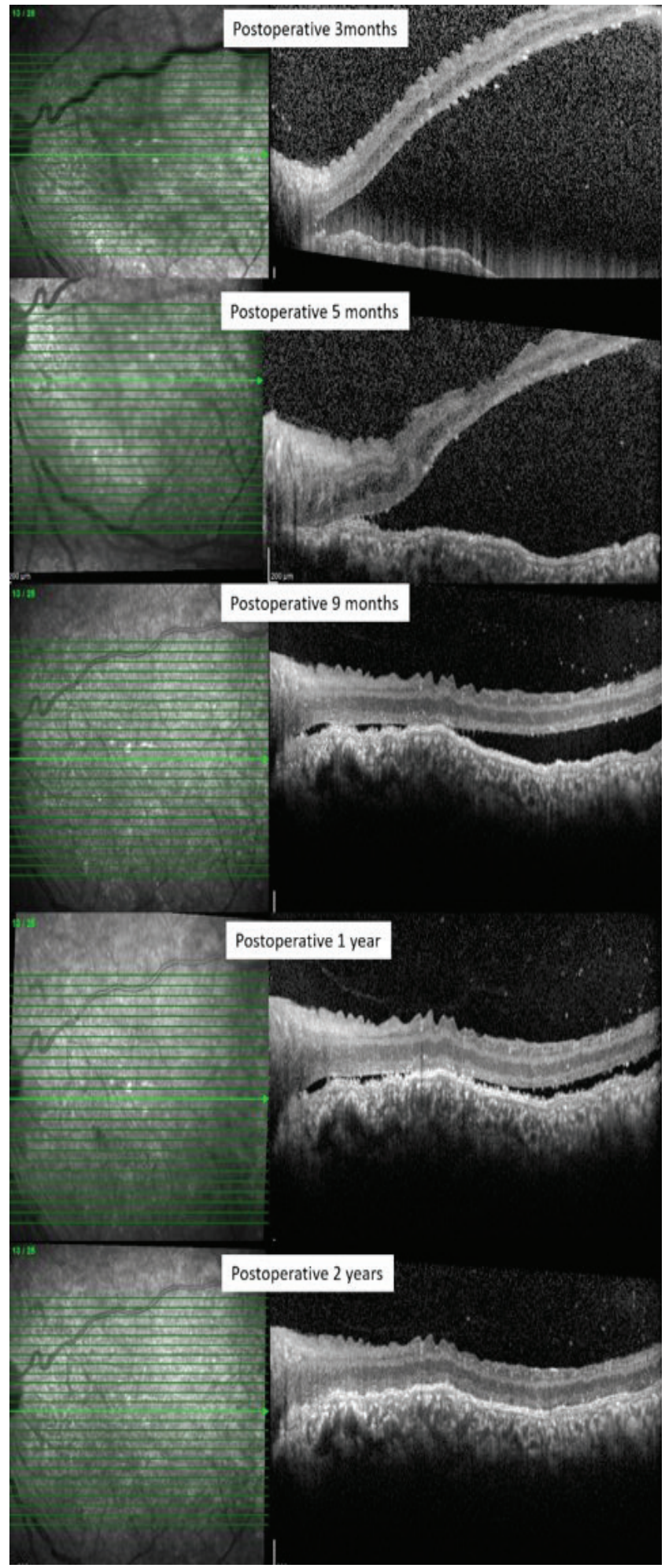

Figure 3. Spectral domain optical coherence tomography scans of the left eye of case 8 revealed gradual resolution of the exudative RD with total reattachment at 2 years

RD: Retinal detachment 

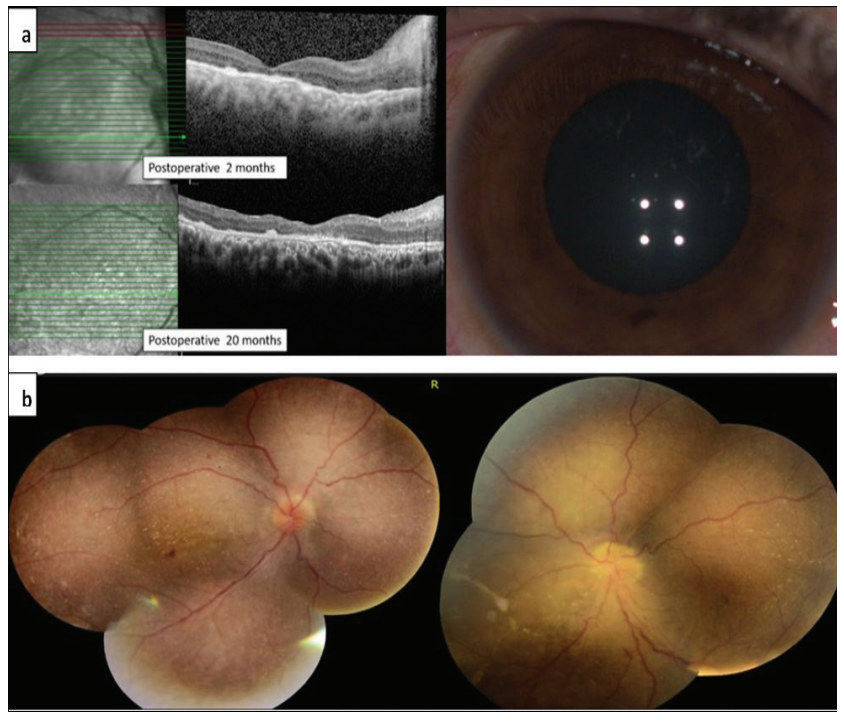

Figure 4. a) Spectral domain optical coherence tomography scans of the right eye of case 8 . Note that the retina was totally reattached following four-quadrant scleral window surgery with drainage of subretinal fluid starting from the first postoperative visit in the right eye and remained attached through the entire follow-up period. b) Fundus images of both eyes of patient 8 showing resolution of the exudative $\mathrm{RD}$ and total reattachment during 20 -month follow-up

$\mathrm{RD}$ : Retinal detachment

\section{Discussion}

In the present study, a final retinal reattachment rate of $78.6 \%$ was achieved, followed by a functional improvement in BCVA in $85.7 \%$ using a modified Gass technique in patients with NO-related UE.

Gass $^{8}$ was the first to suggest the four-quadrant sclerectomy and sclerostomy procedure to treat eyes with UE. He reported total resolution of the supraciliochoroidal and subretinal fluid with this technique within 3 months. Johnson and Gass ${ }^{9}$ reported a series of 23 eyes of 20 patients with idiopathic UE syndrome (UES) operated with a similar technique where UE resolution was seen within 6 months in $96 \%$ of the eyes after one or two procedures, with stable or improved final VA in $91 \%$ of the eyes.

The success rate in the present study was lower than that reported by Johnson and Gass. ${ }^{9}$ This may be because our cases were all extreme NO-related UE in very small eyes (mean AL $15.1 \mathrm{~mm}$ ), in contrast to Gass' series, which were all idiopathic UES. It is important to note that surgery for UE in nanophthalmic eyes has more complications, recurrence, and disappointing results. ${ }^{11,13}$

Many authors have modified the technique in different ways, with varying success rates. Jackson et al. ${ }^{14}$ reported a multicenter series of 10 patients with idiopathic UES treated with a modified Gass technique with deeper sclerectomies (full-thickness or deep enough to visualize the choroid and allow fluid to leak through the sclera), with a $50 \%$ success rate. Uyama et al. ${ }^{15}$ modified the technique to an inferior two-quadrant subscleral sclerectomy with scleral flap and treated 19 eyes of 16 patients with UE. Of the 6 eyes with NO-related UE in their series, reattachment was achieved in $4(66.6 \%)$, and the remaining 2 eyes needed a secondary procedure in the superior quadrants to achieve reattachment, as in cases 6 and 10 in the present series. Another modified technique involving full-thickness sclerotomy without sclerectomy resulted in resolution of subretinal fluid in 4 of 5 eyes with UE, 3 of which were associated with NO. ${ }^{16}$ Mansour et al. ${ }^{17}$ described an extensive circumferential partialthickness (90\% depth) scleral excision technique very similar to our technique in 8 eyes of 5 patients with NO-related UE and reported rapid resolution of the effusion. Intravitreal anti-VEGF injection was also suggested recently to increase the efficacy of partial-thickness sclerectomy surgery for intractable UES. ${ }^{18}$

Recurrences and repeated surgeries for UE have been reported due to sclerectomy closure. ${ }^{13,17}$ Johnson and Gass ${ }^{9}$ reported recurrences in $23 \%$ of their cases, all of which resolved spontaneously or with an additional operation to remove scar tissue from the sclerectomy sites and reopen the sclerostomies. Jackson et al. ${ }^{14}$ reported a total of 19 operations in 14 eyes with idiopathic UES, where 4 eyes required multiple operations. It was suggested that episcleral and scleral scarring might lead to closure of the scleral windows after surgery, and topical application of MMC might prevent such scarring at the sclerectomy site. ${ }^{12,19}$ We suppose that episcleral scarring was the cause of recurrence of macular detachment in case 3 in the present study. However, a second surgery with MMC did not help to control the macular UE in this case. Our hypothesis for the limited response in this patient was the lack of healthy RPE to pump subretinal fluid out because of the pigmentary retinopathy.

The treatment of NO-related UE can be difficult. It is suggested that choroidal inflammation might be an underlying reason for UE. ${ }^{20}$ However, the results of studies on corticosteroid therapy for the treatment of UE are not promising. ${ }^{7,21,22}$ Other medical therapies such as topical non-steroidal anti-inflammatory drugs, topical prostaglandin analogs, and systemic carbonic anhydrase inhibitors have been reported only in a few case reports. $^{22,23}$

It is well known that glaucoma is very prevalent in eyes with NO, most commonly angle-closure glaucoma with progressive shallowing of the anterior chamber that narrows the angle and causes pupillary block. ${ }^{24}$ The incidence of angle-closure glaucoma in NO was reported to be $69.2 \%$ in a high-hyperopia database. ${ }^{25}$ In the management of angle closure in these eyes, laser iridectomy and argon-laser peripheral iridoplasty can be performed. When laser treatments are ineffective, glaucoma filtering surgeries may be needed. ${ }^{24}$

When surgical treatment for UE is needed in nanophthalmic eyes, it may be logical to preserve the superior quadrants for future possible glaucoma surgeries, especially when there 
is already known glaucoma. We believe that two-quadrant sclerectomies can yield successful results in early, mild cases with limited peripheral UE and longer AL (as in case 5). However, it may not be sufficient in severe cases with bullous extensive UE, where it may be necessary to complete the surgery in all four quadrants to obtain the same favorable outcome, as in cases 6 and 10 in this study.

External transscleral subretinal fluid drainage is usually avoided in these cases due to fear of complications like choroidal hemorrhage during choroid puncture because the choroid may be highly congested secondary to blocked transscleral drainage caused by the thickened sclera in these eyes. However, we tried this in the most severe case (patient 8, right eye), in which the retina was behind the lens and vision was at the level of light perception. Although expectations were extremely low, very favorable and unexpected anatomical and functional outcomes were achieved. This approach was recently described in two other cases in the literature, with similar results and no complications. ${ }^{26}$ Therefore, external drainage of subretinal fluid can be performed in addition to sclerectomies for very advanced, highly bullous UE and might lead to a quicker and better response.

\section{Study Limitations}

A limitation of our study was the small number of cases, especially in the two-quadrant surgery group, which is basically because of the low overall incidence of $\mathrm{NO}$ and associated UE with glaucoma. However, strengths of the study were the unique modified technique, being the largest single-center study on the surgical treatment of NO-related UE with relatively long-term follow-up, and the emphasis on two-quadrant surgery to preserve the superior quadrants for future possible glaucoma surgeries in eyes with glaucoma.

\section{Conclusion}

In conclusion, scleral thinning surgeries are effective for the treatment of NO-related UE. Our results supported the effectiveness of the four-quadrant extensive sclerectomy technique. However, two-quadrant surgery might be successful in mild cases with glaucoma to preserve the superior quadrants for future glaucoma surgery. External transscleral drainage of subretinal fluid may be an option in severe cases to achieve quicker resolution.

Ethics

Ethics Committee Approval: Gazi University Faculty of Medicine Clinical Research Ethics Committee (date: 07.12.2020, decision no: 821 )

Informed Consent: Obtained.

Peer-review: Externally and internally peer reviewed.

\section{Authorship Contributions}

Surgical and Medical Practices: Ş.Ö., M.C.Ö., M.H., Concept: Ş.Ö., D.Y.Y., Design: Ş.Ö., Data Collection or Processing: Ş.Ö.,
D.Y.Y., M.H., M.C.Ö., Analysis or Interpretation: Ş.Ö., M.C.Ö., Literature Search: D.Y.Y., M.H., Writing: M.H., D.Y.Y.

Conflict of Interest: No conflict of interest was declared by the authors.

Financial Disclosure: The authors declared that this study received no financial support.

\section{References}

1. Day AC, MacLaren RE, Bunce C, Stevens JD, Foster PJ. Outcomes of phacoemulsification and intraocular lens implantation in microphthalmos and nanophthalmos. J Cataract Refract Surg. 2013;39:87-96.

2. Shah SP, Taylor AE, Sowden JC, Ragge NK, Russell-Eggitt I, Rahi JS, Gilbert CE. Anophthalmos, microphthalmos, and typical coloboma in the United Kingdom: a prospective study of incidence and risk. Invest Ophthalmol Vis Sci. 2011;52:558-64.

3. Hoffman RS, Vasavada AR, Allen QB, Snyder ME, Devgan U, Braga-Mele $\mathrm{R}$ and Committee ACC. Cataract surgery in the small eye. J Cataract Refract Surg. 2015;41:2565-2575.

4. Singh OS, Simmons RJ, Brockhurst RJ, Trempe CL. Nanophthalmos: a perspective on identification and therapy. Ophthalmology. 1982;89:10061012.

5. MacKay CJ, Shek MS, Carr RE, Yanuzzi LA, Gouras P. Retinal degeneration with nanophthalmos, cystic macular degeneration, and angle closure glaucoma: a new recessive syndrome. Arch Ophthalmol. 1987;105:366-371.

6. Elagouz M, Stanescu-Segall D, Jackson TL. Uveal effusion syndrome. Surv Ophthalmol. 2010;55:134-145.

7. Shields CL, Roelofs K, Di Nicola M, Sioufi K, Mashayekhi A and Shields JA. Uveal effusion syndrome in 104 eyes: Response to corticosteroids-The 2017 Axel C. Hansen lecture. Indian J Ophthalmol. 2017;65:1093.

8. Gass J. Uveal effusion syndrome: a new hypothesis concerning pathogenesis and technique of surgical treatment. Trans Am Ophthalmol Soc. 1983;81:246.

9. Johnson MW and Gass JDM. Surgical management of the idiopathic uveal effusion syndrome. Ophthalmology. 1990;97:778-785.

10. Ozgonul C, Dedania VS, Cohen SR and Besirli CG. Scleral surgery for uveal effusion. Retina. 2017;37:1977-1983.

11. Areiter E, Neale M and Johnson SM. Spectrum of angle closure, uveal effusion syndrome, and nanophthalmos. J Curr Glaucoma Pract. 2016;10:113.

12. Akduman L, Adelberg DA and Del Priore LV. Nanophthalmic uveal effusion managed with scleral windows and topical mitomycin-C. Ophthalmic Surg Lasers. 1997;28:325-327.

13. Morita H, Funata M, Kusakari T, Yoshino Y, Kiyosawa M. Recurrence of nanophthalmic uveal effusion. Ophthalmologica. 1993;207:30-36.

14. Jackson TL, Hussain A, Morley AM, Sullivan PM, Hodgetts A, El-Osta A, Hillenkamp J, Charles SJ, Sheard R and Williamson TH. Scleral hydraulic conductivity and macromolecular diffusion in patients with uveal effusion syndrome. Invest Ophthalmol Vis Sci. 2008;49:5033-5040.

15. Uyama M, Takahashi K, Kozaki J, Tagami N, Takada Y, Ohkuma H, Matsunaga H, Kimoto T, Nishimura T. Uveal effusion syndrome: clinical features, surgical treatment, histologic examination of the sclera, and pathophysiology. Ophthalmology. 2000;107:441-449.

16. Kong M, Kim JH, Kim SJ, Kang SW. Full-thickness sclerotomy for uveal effusion syndrome. Korean J Ophthalmol. 2013;27:294-298.

17. Mansour A, Stewart MW, Shields CL, Hamam R, Fattah MA, Sheheitli H, Mehanna C-J, Yassine S, Chahine H, Keaik M. Extensive circumferential partial-thickness sclerectomy in eyes with extreme nanophthalmos and spontaneous uveal effusion. Br J Ophthalmol. 2019;103:1862-1867.

18. Guo J, Cao X and Li X. Partial thickness sclerectomy and intravitreal antiVEGF therapy for intractable uveal effusion syndrome. Int Ophthalmol. 2019;39:1885-1890.

19. Sabrosa NA, Smith HB and MacLaren RE. Scleral punch method with topical mitomycin $\mathrm{C}$ for safe revision of failed deep sclerectomy in nanophthalmic 
uveal effusion syndrome. Graefes Arch Clin Exp Ophthalmol. 2009;247:9991001.

20. Kumar A, Kedar $S$ and Singh RP. The indocyanine green findings in idiopathic uveal effusion syndrome. Indian J Ophthalmol. 2002;50:217.

21. Brockhurst RJ. Vortex vein decompression for nanophthalmic uveal effusion. Arch Ophthalmol. 1980;98:1987-1990.

22. Tong B, Wang C and Qi X. Unusual rapid resolution of postsclerectomy exudative retinal detachment with topical NSAIDs therapy in a case of nanophthalmos. J Int Med Res. 2019:0300060519847376.

23. Derk BA, Benćić G, Ćorluka V, Geber MZ and Vatavuk Z. Medical therapy for uveal effusion syndrome. Eye. 2014;28:1028-1031.
24. Burgoyne C. Nanophthalmia and chronic angle-closure glaucoma. J Glaucoma. 2002;11:525-528.

25. Relhan N, Jalali S, Pehre N, Rao H, Manusani U and Bodduluri L. Highhyperopia database, part I: clinical characterisation including morphometric (biometric) differentiation of posterior microphthalmos from nanophthalmos. Eye. 2016;30:120-126.

26. Khatri A, Singh S, Joshi K and Kharel M. Quadrantic vortex vein decompression with subretinal fluid drainage for manangement of Nanophthalmic choroidal effusions-a review of literature and case series. BMC Ophthalmol. 2019;19:210. 